\title{
Challenges for nanomechanical sensors in biological detection
}

\author{
Montserrat Calleja*, Priscila M. Kosaka, Álvaro San Paulo and Javier Tamayo \\ Received (in $X X X, X X X)$ Xth $X X X X X X X X X 20 X X$, Accepted Xth XXXXXXXXX 20XX \\ DOI: 10.1039/b000000x
}

\begin{abstract}
5 Nanomechanical biosensing relies on changes in the movement and deformation of micro and nanoscale objects when they interact with biomolecules and other biological targets. This field of research has provided ever-increasing records in label-free detection sensitivity but it has not yet been established as a practical alternative for biological detection. We analyze here the latest advancements in the field, along with the challenges remaining for nanomechanical biosensors to become a commonly used tool in biology 10 and biochemistry laboratories.
\end{abstract}

\section{Introduction}

Only six years after the pioneering works by Barnes et $\mathrm{al}^{1}$ and Thundat et $\mathrm{al}^{2}$ proposing microcantilevers as a new class of 15 sensors for a wide range of applications; Fritz and co-workers ${ }^{3}$ were able to discern DNA sequences thanks to an small array of cantilevers ( 8 sensors) and using some of them as reference. Over the last twelve years after this successful detection of non-labeled DNA targets by cantilevers, nanomechanical sensing has 20 provided important advancements in the detection of biomolecules and other biological targets, such as viruses and bacteria. Still, nanomechanical sensing has not yet been established as a practical alternative to well established bioanalytical techniques such as ELISA, microarrays or 25 electrophoresis methods. We discuss here the challenges remaining for nanomechanical biosensors to become a commonly used tool in biology laboratories.

The application range of nanomechanical sensors has broadened in the last years, ranging from gas detection ${ }^{4}$, 30 calorimetry $^{1,5-7}$, drug screening ${ }^{8}$, genetics $^{3,9}$, proteomics $^{10-12}$, microbiology ${ }^{13,14}$, glycomics ${ }^{15}$ and metabolic measurements at the level of individual cells ${ }^{16}$, to cite just a few. A review of the chemicals and bioanalytes already detected by cantilever sensors states that nearly 50 analytes have been successfully detected ${ }^{17}$. A 35 comprehensive analysis of the wide range of applications for nanomechanical sensors is given in previous reviews ${ }^{18}$.

This review focuses on nanomechanical sensing for biological applications, also excellently reviewed previously ${ }^{19,20}$. Other previous review articles related to this field focus on operation 40 modes and read-out methods ${ }^{18}$ or in establishing comparative analysis with other biosensing technologies ${ }^{20}$. Also, the fabrication approaches and materials employed to obtain the micro and nanostructures that are the basis of nanomechanical biosensors have already been recently revised ${ }^{21}$. In addition, some 45 of the fundamental aspects behind nanomechanical sensing have been thoroughly reviewed by Eom et $\mathrm{al}^{22}$.

Nanomechanical biosensors can be roughly classified according to its basic device functionality. These devices can be used to either i) detect the presence or measure the concentration 50 of a given analyte in solution; ii) measure a particular physical property of the analyte for identification/classification purposes; iii) observe, characterize or analyze a particular biological process. There are multiple parameters that describe the device performance of a nanomechanical biosensor: sensitivity, 55 selectivity, response time, throughput, multiplexing capability and robustness among them. Each parameter becomes more relevant accordingly with the particular device functionality. In this review we do not intend to exhaustively revise nor all the particular biological applications explored so far neither the state 60 of the art on the optimization of all possible device performance characteristics. We rather focus on the most prominent remaining challenges, current constraints and recent breakthroughs that are common to most nanomechanical biosensors regardless of its belonging to any of the categories given above.

65 In section 2 we revise the fundamental working principles and operation modes of nanomechanical biosensors. Then, in section 3 we point out the importance of measurement throughput for any biological sensing application, and discuss recent advances regarding this issue. The important role of the intrinsic variability 70 of biological processes in the analysis of nanomechanical biosensing assays is discussed in section 4 , followed by an examination of the implications of label-free detection for nanomechanical detection in section 5. Section 6 remarks the capability of nanomechanical sensing to observe conformational

75 changes in biological systems. In sections 7, 8 and 9 the current trends and progress in device miniaturization, innovative sensor architectures and the search for new mechanical biomarkers are respectively reviewed.

\section{Operation modes, real-time detection and end- ${ }_{80}$ point assays}


A) Static mode

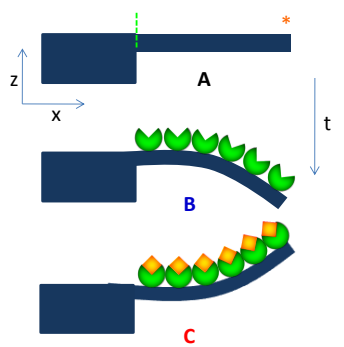

B) Dynamic mode
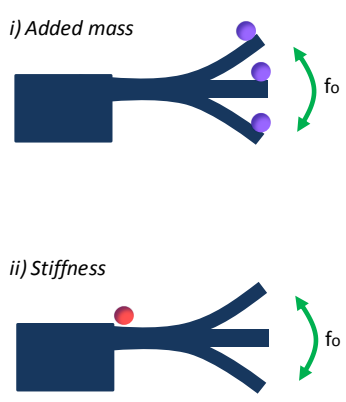
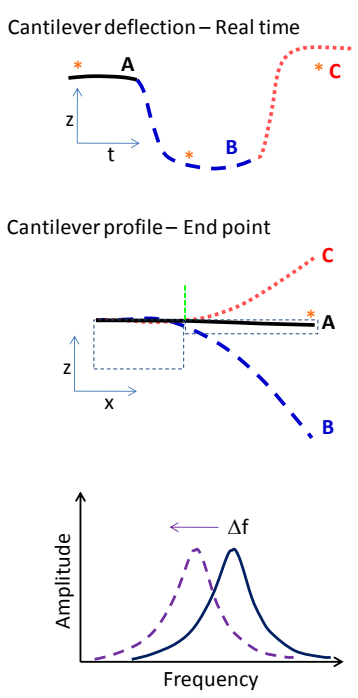

Cantilever profile- End point
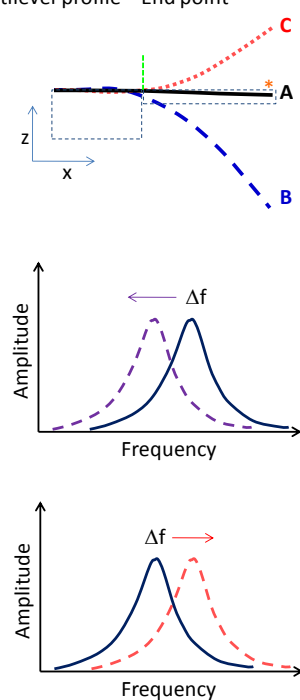

Figure 1. Main operation modes of nanomechanical sensors: static mode (A) and dynamic mode (B). Both modes can operate in real-time or as end-point detection assays. When the static mode is used, measurement of 5 the full cantilever profile, or at least a referenced $\mathrm{z}$ position, is needed for the end-point assay. The dynamic mode can follow changes in the added mass (i) and also changes in the stiffness (ii). When the adsorption is restricted either to the free-end or to the clamped end, both contributions can be disentangled. A practical example can be seen in figure 13 .

10 Nanomechanical sensors can be operated in the static or dynamic mode (figure 1). The resulting mechanical response to the interaction with the biological analyte under test is either a deformation or a resonance frequency shift, respectively. Deformation is normally related to changes in the surface stress 15 arising from intermolecular forces and charge reorganization ${ }^{23}$ and it requires the asymmetric immobilization of bioreceptors, this is, on only one side of the sensor. Resonance frequency shifting happens as a consequence to the added mass of the analyte on any of the sensor surfaces, usually a flexural-mode 20 beam resonator, and in many cases it can also be influenced by changes in the mechanical stiffness of the adsorbate-beam system $^{24}$. By implementation of either an optomechanical or an electromechanical transduction scheme, both the dynamic and the static modes are suitable to produce quantitative measurements 25 that relate the magnitude of the mechanical response of the sensor to the concentration, properties and/or involvement of the analyte in on-going biological processes ${ }^{25-27}$.

Experiments with nanomechanical sensors can be designed to follow the mechanical response in real time or, alternatively, the

30 mechanical signals can be measured before and after incubation of the chips in the test sample to perform end-point detection of an analyte. Detection in real time provides information about the kinetics of the biomolecular interactions, while end-point detection is usually deemed more practical for clinic applications, 35 where the need is limited to detect the presence of an analyte and its concentration.

For the case of the dynamic mode most of the work has been performed in the form of drip and dry end-point detection experiments in air or vacuum ${ }^{18}$, with some valuable exceptions ${ }^{28}$.

40 This is due to the difficulty of the dynamic operation in liquids, arising from the reduced quality factors due to viscous drag with the fluid. In the dynamic mode frequency can be followed after sequential incubation steps. Nanomechanical sensors working in the static or surface stress mode have been commonly used to 45 work in liquid for real-time detection. End-point detection after sequential incubation steps in this mode is elusive to most readout systems, as it is the case for those based in the detection of a single point displacement at the cantilever end. The need to follow the sensor response after each incubation step in end-point 50 assays means that free-end displacements need to be referenced to a fixed point (for example, the clamping chip) or, alternatively, the full profile of the cantilever needs to be measured. This lack of a referenced measurement of the deflection has been overcome by using full field and scanning read-out methods capable of 55 following the profile of the microcantilevers as described in point 3.2. Figure 1A sketches the measurement of the free-end displacement in real-time and the measurement of the full profile of the cantilever after subsequent incubation steps. The capability to perform end-point experiments in the static mode is relevant ${ }_{60}$ for the development of nanomechanical sensors as a routine tool in the clinic. Commonly, sample collection and incubation of biochips are made at distant locations to the place where the testing equipment is based and the possibility to perform ex-situ measurements is a must. Also, one additional appealing 65 advantage of nanomechanical sensing is that measurements can be performed in air as well as in liquid in many cases, which is not accessible to most biosensing technologies.

\section{Throughput and multiplexing capability}

An important bottleneck in the nanomechanical sensing 70 technology lays in the improvement of its throughput. The lack of large datasets acquired with nanomechanical sensors is present in most of the already demonstrated applications. Large numbers of experiments are necessary to push the technology beyond the mere proof of concept. An important step in this direction was 75 undertaken by Fritz and co-workers when they used an array of eight cantilevers to fish for different DNA sequences ${ }^{3}$. Since then, the throughput of nanomechanical biosensing technologies has hardly gone much further. Arrays of eight cantilevers or less are still currently used by scientists worldwide to follow

80 


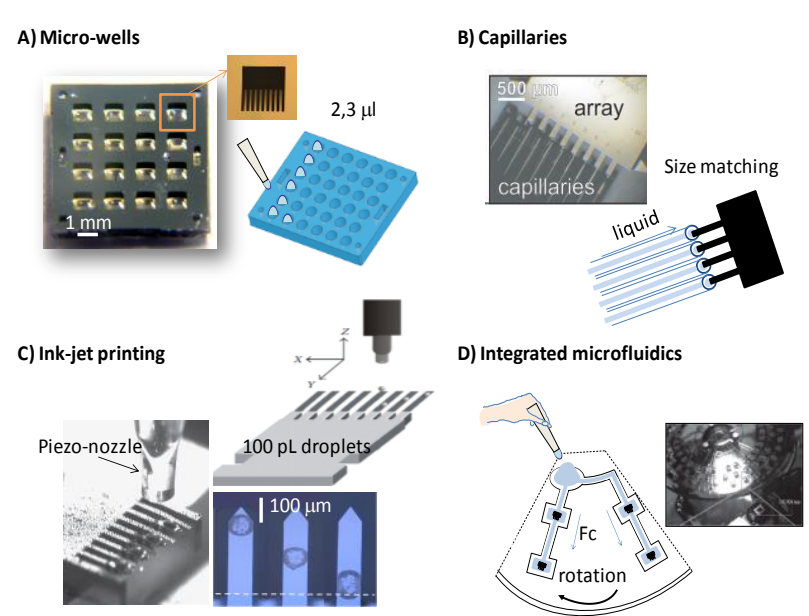

Figure 2. Sample dispensing and surface functionalization strategies for nanomechanical sensors. A) Microwells matching the design of an array of 128 microcantilevers in 16 groups of 8 cantilevers each. B) Capillaries used to individually functionalize 8 cantilevers separated $250 \mu \mathrm{m}$ in an 5 array. C) Optical image of the deposition of microdroplets for cantilever functionalization. This method allows partial wetting of the cantilever surface as seen in the images. D) Integrated microfluidics built in a rotating platform for liquid transport to measurement wells by centrifugal forces. Different parts of the figure are adapted with permission from A) 10 Rev. Sci. Ins. 2010, 81, 125109. Copyright 2010, American Institute of Physics. B) PNAS, 2002, 99, 9783 C) Macmillan Publishers Ltd: Nature Nanotechnology 2009, 4, 179, copyright 2009 and Journal of Sensors 2012, 561256 D) Lab on a Chip, 2011. 11(14), 2411.

15 applications expanding from gas detection to polymer studies and microbiology. Such arrays of few sensors can only provide a limited understanding of processes as complex as DNA immobilization and hybridization, oriented immobilization of antibodies, antigen recognition and site specific or random 20 adsorption of viruses, bacteria or cells.

Recently, increasing efforts to provide the scientific community with massive arrays of nanomechanical sensors have been reported ${ }^{29-31}$, and promising advances on methods to follow their responses in fast, simple and effective manners have also 25 been suggested ${ }^{4,32-35}$. Cantilevers can indeed be mass fabricated in high density arrays, both for the case of passive microcantilevers as those used for optical detection; and active sensors with integrated read-out, as demonstrated for the case of the Millipede probes for data storage ${ }^{36-39}$.

30 The difficulty to attain high throughput experiments with cantilevers arises not only in the sensor fabrication, but also in sensor incubation in diverse test samples and their massive parallel read-out. The same challenge applies to attain high multiplexing capability, with the added difficulty of immobilizing 35 different receptor layers in sensors closely packed into the same array. In principle, nanomechanical sensors have the potential to serve as both high throughput tools for the evaluation of large numbers of samples in a short time; and to provide with high multiplexing capability, so large numbers of different target 40 analytes can be screened per assay. However, this potential has not yet been fully exploited.

\subsection{Functionalization of microcantilever arrays}

Commercial microcantilever chips can be easily managed in serial experiments and the straightforward approach has been the
45 incubation of each single chip containing an array of $2-8$ cantilevers in a microtube. In figure 2 more sophisticated solutions are described for the functionalization of cantilever sensors with receptor biolayers.

Microcapillaries have served to address individually 50 cantilevers in the same array ${ }^{26,40}$. This has allowed $8 \mathrm{X}$ multiplexing and, even more importantly, to use some cantilevers as a reference to filter out unspecific signals. The use of a reference cantilever within the same chip array is preferred, as different chips may contain cantilevers with slightly different

55 mechanical properties which can reduce the effectiveness of the reference signal subtraction method. This approach has proven very efficient when pairs of cantilevers are used, each measurement sensor having its adjacent reference for noise filtering ${ }^{15}$. The drawback in this method is that capillaries spacing 60 must match the pitch dimensions of the array and the sensitization process is time consuming and prone to sensor breaking.

In a different approach, Yue et al used individually addressable reaction wells integrated to the sensor at the fabrication step to follow protease activity and inhibition ${ }^{27}$. In Kosaka et al. a chip 65 containing 16 wells was designed with pitch dimensions matching the SBS (Society for Biomolecular Sciences) standards for compatibility with existing automated dispensing devices. The wells here were also large enough for alternative hand micropipetting of solutions. The design was matching a two70 dimensional cantilever array comprising 128 cantilevers distributed in the 16 reaction wells ${ }^{33,41}$.

More costly, but with the highest potential for the differential sensitization of large arrays, is the ink-jet technology. Ink-jetting is a mature technique used in the fabrication of DNA and protein 75 microarrays. Here, a small droplet of volume of around $100 \mathrm{pL}$ is deposited on the cantilever surface, allowing droplet positioning with an accuracy of tens of micrometers. This method provides with the better flexibility to address sensors of almost any size and design. The main constraint is the rapid drying of the droplet, 80 which limits the incubation time needed to attain the necessarily dense and high quality biolayers. Nevertheless, this drawback can be circumvented by appropriate control of environmental humidity and temperature ${ }^{41}$. Inkjet printing is a promising technology for functionalization because it is fast, versatile and 85 suitable for large-scale integration. This technique suits the need of high-throughput functionalization of arrays of nanomechanical systems as the inkjet head does not contact the surface of the fragile devices. It has already been demonstrated for DNA functionalization of microcantilever arrays ${ }^{43}$.

90 An alternative solution, particularly well suited for real-time experiments, is the fabrication of microfluidics integrated to the sensors $^{27,44,45}$. Bosco et al propose the integration of microfluidics and sensors in a DVD format so the rotation produces the liquid displacement by centrifugal forces to the measurement wells ${ }^{46}$.

\section{3.2 Read-out of microcantilever arrays}

Regarding the read-out of arrays of sensors, electrical read-out is well suited for measurements of large arrays ${ }^{29,38}$. Parallel readout of microcantilever arrays has been achieved by integration of piezoresistive sensors and circuitry in the 100 cantilevers themselves ${ }^{47,48}$ such as MOSFET integration ${ }^{49}$ or by capacitive methods ${ }^{29}$, among other alternatives ${ }^{21}$. The main advantage of this integrated solution is that external alignment is 


\author{
A final version of this manuscript can be found at \\ http://pubs.rsc.org/en/content/articlelanding/2012/nr/c2nr31102j
}

ARTICLE TYPE

A) Phase shifting interferometry

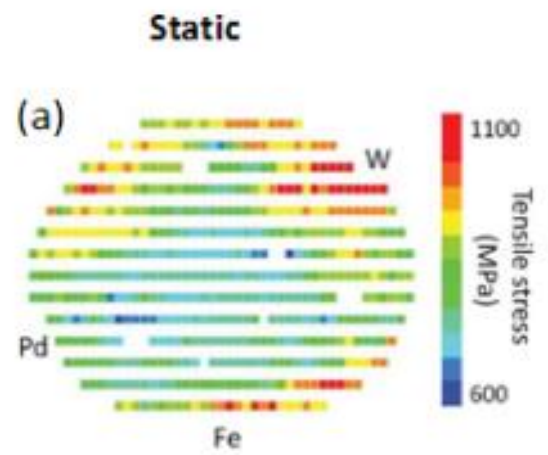

(b)

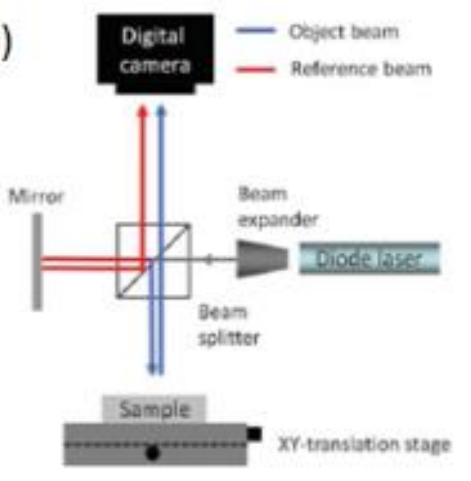

B) Scanning laser analysis

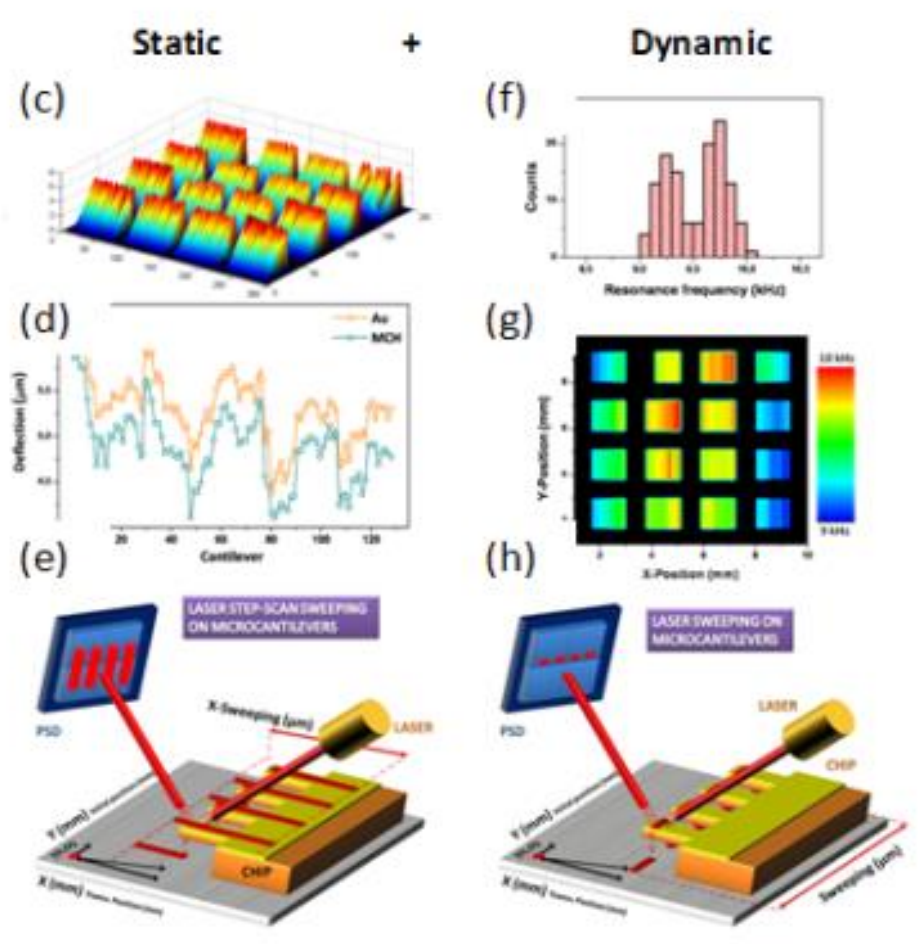

Figure 3. Sketches of the basics of phase shifting interferometry (A) and scanning laser read-out techniques (B) with example results in arrays of sensors for each method. A) (a) Color-coded visualization of stresses of the microcantilevers in a wafer. (b) Scheme of the experimental set-up. Reprinted with permission from Rev. Sci. ins., 2011, 82, 063903. Copyright 2011, American Institute of Physics. B) Scanning laser read-out technique combines the static and dynamic detection in a single experiment. Static (c): three dimensional image of an array of 128 microcantilevers and in (d) displacement of the 5128 microcantilevers before and after incubation with mercaptohexanol. Dynamic: (f) Histogram of the resonance frequency of the 128 cantilevers in (c). (g) Frequencies of each cantilever in the chip plotted as color intensity map. In (e) and (h): the laser trajectories during the measurements. Reprinted with permission from Rev. Sci. Ins. 2010, 81, 125109. Copyright 2010, American Institute of Physics.

unnecessary, although active sensors of this kind are not yet 10 widely available and the fabrication costs are high. Also, electrical read-out has not been extensively used in liquid, given the added difficulty of encapsulation of the sensor circuitry.

Optical read-out has been preferred in biosensing applications 20 for this read-out scheme. AFM heads have been commonly used to implement this method to follow the static and dynamic response of cantilever sensors. Clearly, an AFM head is not best suited for high throughput measurements, as each new experiment requires the manual alignment of the laser beam to 25 the cantilever free end. Also, measurement of the deflection at a single point precludes its application to end-point experiments in the static mode.

The pioneering effort to improve the throughput of the laser leverage technique came with the work by Lang et al. ${ }^{50,51}$ where of nanomechanical sensors. Commonly, a laser beam is focused 15 at the cantilever free end to follow deformations and vibrations of the cantilever through the reflected beam position in a position sensitive photodetector. Passive tipless cantilever arrays fabricated in silicon and silicon nitride that can be purchased from manufacturers of AFM probes at reasonable prices are used 30 an array of VCSELs (Vertical-Cavity Surface-Emitting Lasers) was addressed to the now very popular eight cantilever array. This approach provides convenient parallel read-out and the capability to use some of the sensors as a reference. The main drawback is the limited flexibility imposed by the necessarily 35 fixed spacing of the cantilever sensors and fixed number of devices. Also, only real-time experiments can be performed in the static mode with this solution. An on-chip optical read-out based on integrated waveguides in the cantilever themselves has also proven suitable to follow microcantilever oscillations ${ }^{52,53}$. 
An alternative laser-leveraging read-out was proposed in $2005^{54}$. This is based on mounting either the laser or the sample in voice-coil actuators, so the displacement of the beam on the surface in two dimensions allows to attain a three dimensional 5 picture of the surface and therefore, the deformation of the cantilevers with respect to the clamping chip in different conditions $^{33,41,55,56}$. Examples of such three dimensional images of cantilever arrays can be seen in figures 3 and 4 . This method gives access to static measurements both in end-point and real10 time approaches. It can also provide simultaneous static and dynamic characterization ${ }^{55}$ and image the shape of vibration modes $^{57}$ up to the $22^{\text {nd }}$. Recently, the relative displacement of the laser beam and the surface has also served to read cantilever arrays with a DVD head ${ }^{46}$. Other optical read-out methods 15 suitable for full field characterization of microcantilevers are phase shifting interferometry and white light interferometry, also with no constraints in the device design ${ }^{32,58-61}$. These techniques are best suited to follow the static response, as the process to attain the dynamic response is time consuming. Also, their 20 implementation in liquids is not trivial ${ }^{62}$. Alternatively, laserdoppler vibrometry possesses high potential for dynamic measurements of cantilever arrays; however, it does not provide information about the static deformations ${ }^{63}$.

\section{Sensitivity, specificity and biological noise}

25 Along the last decade, researchers have pursued the miniaturization of nanomechanical devices and the reduction of their noise levels to the thermomechanical noise floor. Although thoroughly understanding the physics behind the nanomechanical detection and improvement of the sensor performance are both

30 key tasks, looking for the perfect nanomechanical biosensor may be sterile if the inherent variability of biological processes is disregarded.

An alternative route is that a detection threshold be defined for nanomechanical sensors in each target application. This requires 35 of the measurement of a significant number of data points, so we can first define the noise level of the system, which will depend on the receptor layer of choice, the background medium, the device intrinsic mechanical properties and surface characteristics, and even in the laboratory environment, such as temperature, 40 relative humidity and the variability of those parameters.

The choice of the measurement threshold will influence the rate of false positives (FP) and true positives (TP) of the analysis, or, related to that: the balance between sensitivity and specificity of the nanomechanical assay. This threshold cannot be wisely 45 decided without a measurement of the variability of the experimental background. A positive control and a negative control should always be included in nanomechanical analysis, as it is common practice in widely used assays such as ELISA or Western-Blott. This is necessary to know the signal output that 50 arises from unspecific interactions and from the complex processes that occur on the surface when analytes, but also undesired molecules, interact with the receptor biolayer.

Conclusions about measurements of biomolecule interactions cannot be derived from few data points, even for the case of ${ }_{55}$ ideally responsive nanomechanical sensors. This is due to the complex behaviour of such biolayer-nanomechanical systems, and ultimately, to the selectivity of the receptor-target pair. In addition, recent studies point at the difficulty of obtaining ideally perfect receptor biolayers even for the case of self-assembled 60 monolayers ${ }^{64}$. Therefore, complex responses should be expected when applying nanomechanical sensors to biological detection.

We must note that efforts to characterize the biological noise in nanomechanical assays came early, as in ref. [13] where 44 nominally identical resonators were used to address the weighing 65 of single viruses. The biological noise in these experiments was measured as 50 attograms, while the detection limit of the sensors was much lower, in the range of 0.1 attograms. Later, in 2008 Yue at al. detected PSA at a concentration of $1 \mathrm{ng} / \mathrm{mL}$ with an array of 900 microcantilevers in the static mode ${ }^{65}$, while the 70 detection limit of their system was better than $0.2 \mathrm{ng} / \mathrm{mL}$.

In most works presented to date, nanomechanical sensors detection limit is much smaller than the biological ground noise ${ }^{13}$, being the limiting factor not the intrinsic sensor responsivity. In most cases, constraints are common to most biosensing platforms 75 available, particularly those based in surface chemistry, as microarrays, SPR and QCM. As suggsted in the review by Arlett $^{20}$, the biological ground noise cannot be ignored nor eliminated. This first arises from specificity of the ligand-receptor pair in the assay. In the surface stress mode, a biological ground 80 noise also comes from the process of surface immobilization and the necessary percolative surface arrangement to attain large stresses, as studied by Sushko et $\mathrm{al}^{66}$. This intrinsic noise should not be ignored, but it should not be perceived as an impediment to attain reliable assays with the nanomechanical principle in the 85 static mode. Most widely used biosensors live with a degree of randomness in the analysis that must be accounted for.

It is useful to use the ROC (Receiver Operating Characteristic) curves from signal detection theory ${ }^{67,68}$ to account for the needed balance between sensitivity and specificity, as it is an intuitive 90 way to pursue the goal of a useful sensor. Here usefulness means that the target sensitivity and specificity may vary regarding the application of intend. This approach is commonly used to analyze the diagnostic value of a given assay and to decide the optimum detection threshold for a given application. Sensitivity is then ${ }_{95}$ defined as the rate of true positives in the assay, $\mathrm{TP} /(\mathrm{TP}+\mathrm{FN})$, and specificity as the rate of true negatives in the controls, $\mathrm{TN} /(\mathrm{TN}+\mathrm{FP})$. The sensitivity and specificity defined in this way depend on the arbitrary selection of a decision threshold or cutoff level. The ROC curve describes the diagnostic value of the 100 test for each given decision threshold depicting the relation between the rate of true positives (sensitivity) and false positives (1-specificity) encountered in the analysis. The closer to the upper left corner, the better it is the diagnostic value.

In the ROC graph the perfect sensor would be at $(0,1)$ 105 coordinates, but most commonly the decision comes from choosing a decision threshold that is restrictive (left side of the graph, figure 4) when a positive classification is only wanted when strong evidence is found; or ample (right side), when detection is made with weaker evidence (higher sensitivity), but 110 the rate of false positives therefore increases (lower specificity). ROC curves usually involve thousands of measurements and controls.

In nanomechanics the technological bottleneck has been that the measurement throughput has been commonly limited, and 115 negative and positive controls have only been used to try to 
define a sensor in the unrealistic $(1,0)$ coordinates. This has led the search for the perfect cantilever array, with sensors of same mechanical properties and dimensions similar down to the nanometer scale. This route is necessary and it will push 5 nanomechanical sensors to superior performance levels but it cannot be the unique path.

At the actual development stage of nanomechanical sensors we must already be able to state the level of specificity and sensitivity of a particular assay. This will of course depend on the 10 bioreceptor layer of choice and the target to be detected. From data in reference [69] a ROC curve can be derived where the

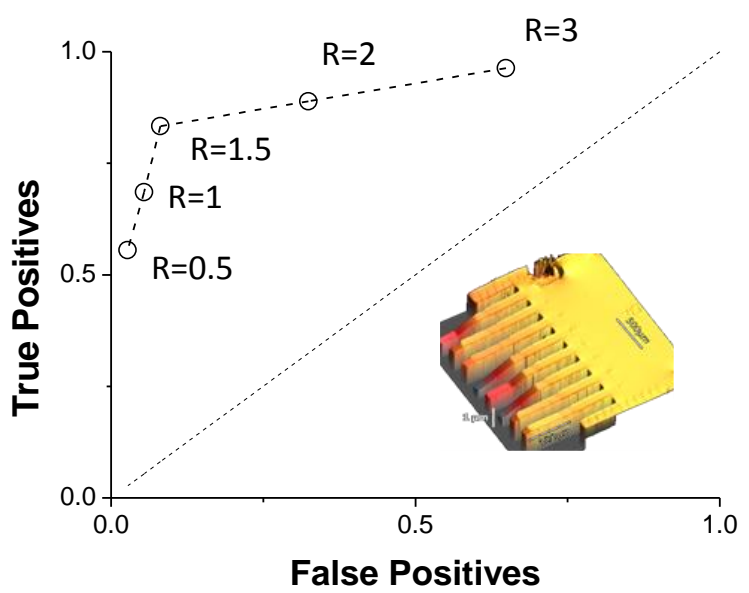

Figure 4. ROC curve for a label-free nanomechanical immunoassay. Detection threshold, $\mathrm{R}$, as defined in the text. The assay studied is the 15 detection of HRP at a concentration of $1 \mu \mathrm{g} / \mathrm{mL}$. The biological noise (false positive rate) was measured using two approaches as control experiments; in the first one an antibody non specific to HRP, antibody against human chronic gonadotropin hormone (anti-hCG, clone 5014) in a concentration of $50 \mu \mathrm{g} / \mathrm{mL}$, was immobilized on the microcantilever 20 surface and, in the second one, the microcantilevers immobilized with the anti-HRP were exposed to lysozyme $(1 \mu \mathrm{g} / \mathrm{mL})$. The curve is the result of studying 120 measurements including detection assays and negative controls. The protocol for immobilization of the receptor layer was previously optimized by analyzing 3364 responses from 841 individual 25 cantilevers.. The protocol was directly applied to the 120 new cantilevers and no further discrimination of defective or non responding cantilevers was made. The inset shows a 3D image of one array of eight sensors obtained by the scanning laser technique (SCALA, Mecwins S.L.). Mechanical defects in some cantilevers in this array are visible.

30 cantilever assay demonstrates a sensitivity of $83 \%$ and a specificity of $92 \%$ for the detection of horseradish peroxidase (HRP). The detection threshold was defined as $R=\left(\sigma_{A g}-\left\langle\sigma_{c}\right\rangle\right) / \Delta \sigma_{c}$, where $\sigma_{A g}$ is the analyte-induced surface stress, $\left\langle\sigma_{c}\right\rangle$ is the mean surface stress obtained in a large 35 dataset of control experiments and $\Delta \sigma_{\mathrm{c}}$ is the standard deviation of the surface stress in the control experiments. Different cut-off values of $\mathrm{R}$ relate to different positions in the ROC curve, as can be seen in figure 4. Here, commercial cantilevers from Concentris $^{40}$ were used and the defect cantilevers at the 40 manufacturing step and the cantilevers with a receptor coating below the quality threshold were not removed from the analysis. This means sensitivity and specificity could be improved further

\begin{abstract}
by a
previous

45 quality

control of

the

functionaliz

ed sensors,

50 in cases

when this

is deemed

practical.
\end{abstract}

Next

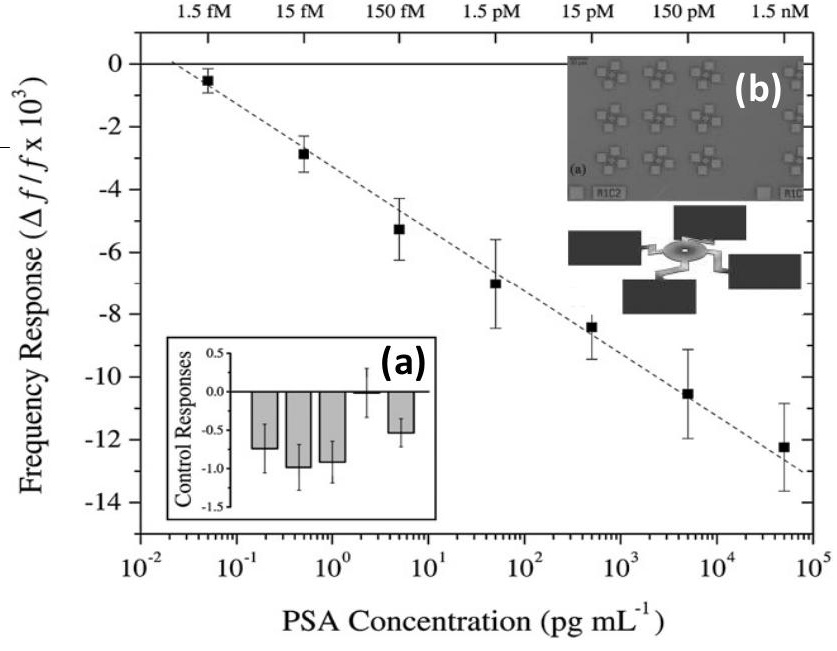

55 step would be to try to avoid measuring hundreds of samples to optimize the threshold value, but this first screening work cannot be avoided nor it is realistic to try to substitute it by the search of the ideal sensor. A proper threshold value found in a large scale screening test and control could be appropriate for subsequent 60 measurements conducted in similar conditions (pre-coating, immobilization density, sensor dimensions, receptor biolayer specificity and similar laboratory environment) with no need for repeating all the controls. But a new large data set test must be performed every time a new application is proposed and when a 65 new biolayer or new experimental conditions are required.

After a full analysis of the experimental background is

Figure 5. Linear dependence of resonance shift with increasing concentration of PSA. Nanoparticles were used here as labels. The inset (a) shows control responses observed during the tests performed at 70 different concentrations, demonstrating consistent but slightly varying background signals due to variations in non-specific binding and environmental conditions from day-to-day. The inset (b) shows the trampoline devices used in the tests. Adapted from Lab Chip, 2009, 9, 3095.

75 performed and given a defined application, the weight can be placed on a particular figure of merit of the biosensor: the sensitivity, the detection time, or the specificity. A home pregnancy test must be fast, a test for VIH should be almost false positive free and an assay targeted to identify new biomarkers 80 should put the stress on sensitivity, even at the cost of a high false positive rate and time consuming processing, otherwise relevant information can be lost. Going back to the ROC curve in figure 4, the optimum threshold value, this is, closer to the $(0,1)$ coordinate, is $\mathrm{R}=1.5$. However, it could be advantageous for a 85 certain application to set it to $\mathrm{R}=2$ to attain better sensitivity at the cost of lower specificity, or, on the contrary, to set $\mathrm{R}=0.5$, for a high specificity at lower sensitivity. Actually, the ability to easily tune the position in the ROC curve is an additional merit of a biosensor.

90 This interplay among different figures of merit of a biosensor infers the extreme difficulty in comparing different technologies. One of the best documented comparisons of nanomechanical sensors to other techniques is given in reference [20], where the figures of merit considered are the limit of detection and the 95 analysis time, both relevant parameters for many applications.

\section{Revising the label-free detection attribute}

The first successful cantilever sensing method, static assays, do not specially benefit from further labelling, and most measurements in the static mode are label-free. This has 
historically put the nanomechanical sensors as an example of label-free biosensors. Nevertheless, the case for nanomechanical dynamic biodetection is different. Despite dynamic nanomechanical sensors have proven good performance without 5 labels; labelling can largely improve specificity and it can lower the detection limit. Therefore, one of the key attributes of nanomechanical biosensing, being a label-free technique, could be revised in many relevant applications, where the cost of eliminating a labelling step can be too detrimental in specificity.
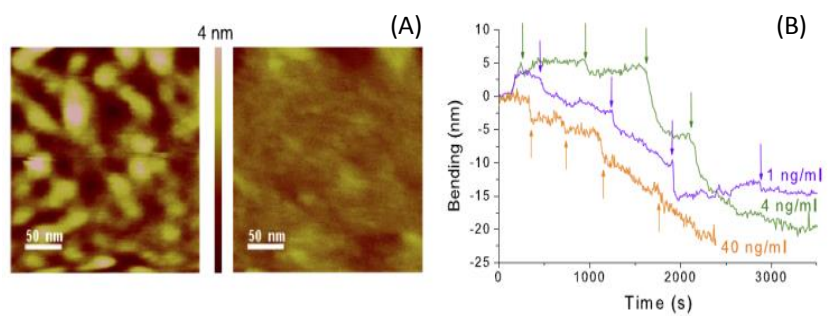

10 Figure 6. ATP-induced conformational changes in the terminase of bacteriophage T7 (gp19). (A) AFM topographic image of the terminases at $40 \mathrm{ng} / \mathrm{mL}$ before (left image) and after ATP interaction (right image) showing the disassembling process of the gp19 oligomers. (B) Cantilever bending upon interaction with ATP measured for different concentrations 15 of terminases: $40 \mathrm{ng} / \mathrm{mL}$ (orange), $4 \mathrm{ng} / \mathrm{mL}$ (dark green) and $1 \mathrm{ng} / \mathrm{mL}$ (violet). The first four observed transitions are marked with an arrow. The measurements indicate a stepped behavior visible up to $1 \mathrm{ng} / \mathrm{mL}$. ATPinduced conformational changes in the terminase of bacteriophage $\mathrm{T} 7$ (gp19). (A) AFM topographic image of the terminases at $40 \mathrm{ng} / \mathrm{mL}$ before

20 (left image) and after ATP interaction (right image) showing the disassembling process of the gp19 oligomers. (B) Cantilever bending upon interaction with ATP measured for different concentrations of terminases: $40 \mathrm{ng} / \mathrm{mL}$ (orange), $4 \mathrm{ng} / \mathrm{mL}$ (dark green) and $1 \mathrm{ng} / \mathrm{mL}$ (violet). The first four observed transitions are marked with an arrow. The

25 measurements indicate a stepped behavior visible up to $1 \mathrm{ng} / \mathrm{mL}$.

Labelling of samples for nanomechanical detection has demonstrated advantageous in end-point assays in the dynamic mode. Craighead and co-workers demonstrated that by labelling with nanoparticles a monoclonal antibody in a sandwich 30 immunoassay they were able to improve the limit of detection by three orders of magnitude to reach $2 \mathrm{ng} / \mathrm{mL}$ in the detection of prion proteins ${ }^{12}$ and even the presence of $50 \mathrm{fg} / \mathrm{mL}$ of PSA spiked in foetal bovine serum background ${ }^{25}$. The technique is also quantitative, as the authors found a clear linear dependence of the 35 frequency response with PSA concentration (figure 5). The ability to detect $\mathrm{fM}$ concentrations of a protein target in a realistic background places labelled resonant cantilever sensors in an excellent position to compete with well established techniques ${ }^{20,70}$.

\section{${ }_{40} 6$. Conformational changes and the surface stress response}

In addition to their capability to detect binding events and adsorbate masses, cantilevers have been successfully used to follow dynamic processes such as conformational changes.

${ }_{45}$ Ghatkesar et $\mathrm{al}^{71}$ followed lipid vesicle $(\sim 748 \times 106 \mathrm{Da})$ adsorption on microcantilevers and their structural rearrangement upon binding of the bee venom peptide melittin (2840 Da) to the vesicles. They measured simultaneously the dynamic response and the static bending in liquid, in order to follow molecular ${ }_{50}$ binding and also the subsequent effect on the biological system in vitro. Conformational changes in DNA molecular motors fuelled by protons have also been studied ${ }^{72}$. Recently, the interaction of a SAM of 16 mer ssDNA and the trivalent spermidine cation has been followed by cantilevers and the results give direct evidence 55 that trivalent ions turn the repulsive electrostatic forces between short strands of single stranded DNA into attractive, as a previous step to condensation ${ }^{73}$. The follow up of DNA films upon changes in relative humidity ${ }^{74}$ had also shown before the presence of attractive forces for highly packed SAMs, recently 60 modelled by Rabin et al. ${ }^{75}$. Also, cantilevers have been able to measure for the first time in real-time ATP-induced conformational changes in the terminase of bacteriophage $\mathrm{T} 7$ (gp19). The recording of the cantilever bending during its functionalization shows the existence of a gp19 monolayer 65 arrangement confirmed by atomic force microscopy of the immobilized proteins (Figure 6). The ATP hydrolysis of the gp19 terminase generates a stepped motion of the cantilever and points to a mechanical cooperative effect among gp19 oligomers ${ }^{76}$.

These are just some examples of relevant biological analysis 70 not accessible to any other available technique. These results point at microcantilever sensors as a unique tool to follow structural changes in biological systems. Conversely, biolayers have served to actuate microcantilevers. As an example, Eom et al have recently attained reversible nanomechanical actuation of a 75 microcantilever driven by light induced conformational changes of i-motif DNA chains ${ }^{77}$.

\section{Miniaturization trends in nanomechanical biosensing}

Miniaturization of nanomechanical biosensors is probably the 80 most suggestive way to either push their performance characteristics to their fundamental limits or to explore new functional concepts. Although cantilever sensors with reduced dimensions are suitable for static detection with improved sensitivity, miniaturization is particularly significant for the

85 dynamic mode because the fundamental properties of a resonant nanostructure are greatly influenced by scaling-laws, thus resulting in an extreme or completely different behaviour deep down the nanometer scale.

Biomolecular spectrometry based on nanomechanical mass 90 sensing represents the paradigm of the benefits of device miniaturization. The perspective of a nanomechanical mass spectrometer with single-atom resolution has motivated strong efforts towards diminishing the inertial mass of sensors based on nanomechanical resonators whose resonance frequency shifts in 95 response to the adsorption of biological entities such as viruses, proteins or oligonucleotides. Figure 7 shows a chronological sequence of mass detection limit achievements compared to the corresponding device effective mass. The most recent advances in nanomechanical mass biosensing include the detection of a 100 single virus ${ }^{13}$ and the weighing of proteins ${ }^{78}$.

Although miniaturization of mass sensors for biomolecular spectrometry has been pursued from different approaches, including the application of vibrating nanomembranes ${ }^{79}$ inspired in cryogenic microcalorimeters developed a decade ago $^{80}$; 105 semiconductor nanowires (NWs) and carbon nanotubes (CNTs) are receiving an increasing interest as active components of the ultimate nanomechanical mass sensors ${ }^{81-83}$. Detection limits 
approaching the mass of a single atom have already been attained $^{84}$ and extreme downsizing of CNT devices has recently allowed inorganic mass detection down to the yoctogram scale by the fabrication of resonators with an effective mass in

5 the range of $300 \mathrm{zg}^{85}$. However, how this trend can impact biosensing is still difficult to predict. Though a high mass resolution is desirable in a nanomechanical biosensor in order to distinguish biomolecules with similar masses, the sensor must also be able to weigh biomolecules with a relatively large mass, 10 which imposes a lower limit for the effective mass of the sensor

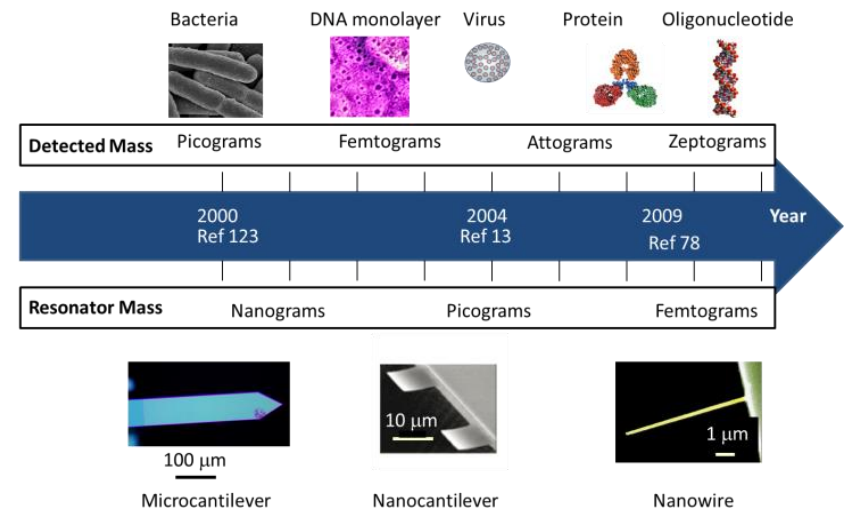

Figure 7. Examples of the detection of different bioanalytes by nanomechanical sensors in the dynamic mode. Below the arrow we note the mass of the sensor able to attain the limits of detection to target a given analyte mass. The years in the arrow approximately mark when a 15 record in mass detection was achieved.

depending on the target molecule. For instance, antibody molecules have masses in the range of $100 \mathrm{zg}$, so that a sensor effective mass in the range of 10 ag with a mass resolution in the sub-zg scale would be desirable. Such compromise between mass 20 resolution and mass detection range can be obtained by maximizing the frequency stability of the device, and recent works have pointed out different approaches in such direction, such as current annealing of CNT resonators ${ }^{85}$ or optomechanical self-excitation of Si nanowire resonators ${ }^{86}$.

25 But exploiting the exceptional detection limits of miniaturized nanomechanical biosensors faces further important challenges. Their practical application would require the integrated fabrication and readout of a large number of devices. Though significant advances in the large-scale integration of bottom-up 30 nanostructures into functional devices has been achieved ${ }^{87}$, in the long term it can be expected that top-down fabrication technologies provide an ideal compromise between miniaturization and large-scale integration, as well as to enable significant improvements in efficient read-out signal 35 amplification via CMOS-integration of the nanomechanical sensors ${ }^{88}$ for the case of electrical read-out. Another non-trivial issue is matching a particular transduction scheme with a sample delivery method that enables to transport the biomolecules under test to the sensitive area of the sensor device. Matrix-assisted 40 laser desorption/ionization (MALDI) and electrospray ionization (ESI) are well-established procedures currently used in combination with time-of-flight (TOF) mass analyzers for protein spectrometry. Although these methods are potentially compatible with nanomechanical detection ${ }^{34,78,79}$, their practical 45 implementation will require high efficiency in terms of the fraction of analyte molecules reaching the detector, which brings back the need of large-scale device integration into sensor arrays that maximize the active sensing area.

Along with these challenges, miniaturization brings out new 50 opportunities regarding novel detection and transduction concepts relevant to biosensing. The unique physical properties of nanomechanical structures when their dimensions are pushed down the nanometer-scale can be exploited to develop novel transduction schemes that further improve the performance of 55 nanomechanical biosensors. For instance, doubly clamped Si nanowire beams ${ }^{89}$ can show extraordinarily high piezoresistive

Figure 8. (a) Photograph of an array of fifteen coupled cantilevers with a $5 \mu \mathrm{m}$ diameter borosilicate microsphere attached to cantilever 14 (inset). (b) Representation of the measured sixth mode of the array before (left) 60 and after (right) the addition of a $5 \mu \mathrm{m}$ microsphere to cantilever 14. In this case, the vibrations localized in cantilevers 14 and 15 after the sphere was added. Reprinted with permission from Appl. Phys. Lett. 92, 114102 (2008). Copyright 2008, American Institute of Physics.

coefficients that enable an integrated read-out of their high ${ }_{65}$ frequency vibrations by measuring the change in electrical

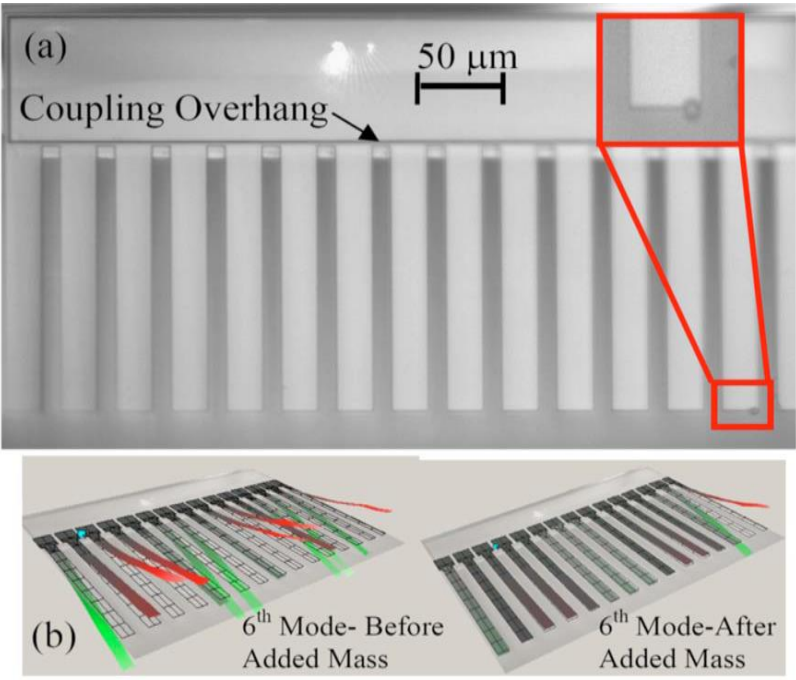

resistance as they vibrate ${ }^{90}$. These nanostructures have also been read-out optically and they have revealed the role of optical resonances associated to light confinement in subwavelength structures to either cool the mechanical motion to $30-40 \mathrm{~K}$ or to 70 enhance the quality factor to values near one million ${ }^{86}$. Another area of impact of miniaturization under exploration is the improvement of the dynamic range. Resonant nanostructures suffer from naturally low dynamic range because their high aspect ratio makes that their oscillation amplitudes are 75 comparable to their diameter/thickness. In consequence, their driven oscillations easily fall into the nonlinear regime. However, different approaches have been reported to either counteract ${ }^{91}$ or to exploit ${ }^{92}$ the nonlinear oscillations of nanomechanical structures.

80 Finally, it should be pointed out that some particular biosensing applications may not be suited to obtain any advantage from the miniaturization of the sensing device. For instance, the final goal in some cases is not detecting a few biomolecules, but rather obtaining a highly distributed mass 85 sensitivity, as demonstrated by the successful detection of picomolar concentrations with large cantilevers ${ }^{93-95}$ and bulk disk 
resonators ${ }^{96}$ in a similar way to the quartz crystal microbalance $(\mathrm{QCM})^{97}$.

\section{New architectures for nanomechanical detection}

The most widely used design for nanomechanical sensors is a 5 cantilever. Similar to this AFM inspired classic design, also doubly clamped beams and the shrink version of these, string resonators have been used ${ }^{21}$. Slight variations of this basic design have been successfully implemented, as the trampoline resonators seen in figure $5^{25}$. This simple and efficient design is nevertheless 10 not the only one available, nor always the best suited for a particular biological application. We revise here alternative architectures and new paradigms for nanomechanical biosensing.

\subsection{Coupled oscillators}

The performance of sensors based on resonant cantilevers 15 could be significantly enhanced by using an array of elastically coupled nanomechanical resonators of identical size. As discussed above, the filtering of the biological noise is a challenge in nanomechanical sensing and coupled oscillators are capable of filtering unspecific signals naturally. In these systems,

20 each individual resonance frequency for a single cantilever system splits into $\mathrm{N}$ frequencies for the array of $\mathrm{N}$ coupled cantilevers (Figure 8), and elastic waves easily propagate through the array. The rich and complex dynamics of these systems opens the door for new devices based on cross-correlation 25 measurements with superior signal to noise ratios. In addition, the collective behaviour of these arrays exhibit new phenomena such as intrinsic localized modes and solitons that can give rise to new sensing paradigms. The vibration localization due to mass disorder was first used by Spletzer et $\mathrm{al}^{63,98}$ to develop a mass 30 sensor consisting of a pair of elastically coupled cantilevers where mass adsorption occurs on one of the cantilevers. Using a pair of elastically coupled cantilevers which were three orders of magnitude smaller in size than those in previous studies, GilSantos et $\mathrm{al}^{99}$ proposed to monitor the change in the ratio of the 35 resonance amplitudes of symmetric and antisymmetric modes measured on only one of the cantilevers, to determine the adsorbed mass. With this method, initial disorders, i.e. differences between coupled sensors, are no longer required to be near zero, thus removing a huge obstacle in the further 40 development and miniaturization of coupled resonators. Moreover, the need of calibration of the amplitudes was eliminated. The same authors have shown that the sensitivity of mass sensors based on the vibration localization phenomena in coupled cantilevers can be alternatively enhanced by decreasing 45 the coupling constant with no need of making the cantilevers smaller, as it occurs when the mass is detected via the resonance frequency measurement ${ }^{100}$. In particular, the coupling constant of pairs of cantilevers elastically coupled by an overhang exponentially decreases with the separation between cantilevers, 50 being the mass sensitivity inversely proportional to the coupling constant. They were able to detect $170 \mathrm{fg}$ of attached mass. Thiruvenkatanathan et al. determine the detection limits of this new paradigm and they show they can largely surpass the responsivity of single resonators ${ }^{101}$. Pakdast et al have recently 55 applied the measurement of amplitude variation to three coupled cantilevers $^{102}$. These recent advancements pave the way to further develop this new paradigm for biochemical detection.

\subsection{Suspended microchannel resonators}

When the aim is the detection of whole bacteria or even ${ }_{60}$ biomolecules in their native environment, one problem in nanomechanical resonators is the viscous drag with the media, which limits the quality factor, $\mathrm{Q}$, and therefore the minimum significant frequency shift. Although some improvement can be made by using higher vibration modes ${ }^{28,103}$, very high Qs in 65 liquid are difficult to attain.

Burg et al have circumvented this limitation by driving the liquid inside the resonator, thus the device can be encapsulated in vacuum for optimum $Q$. This suspended microchannel resonators

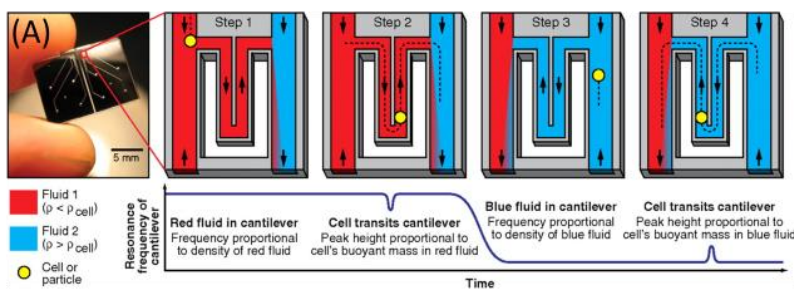

(B)

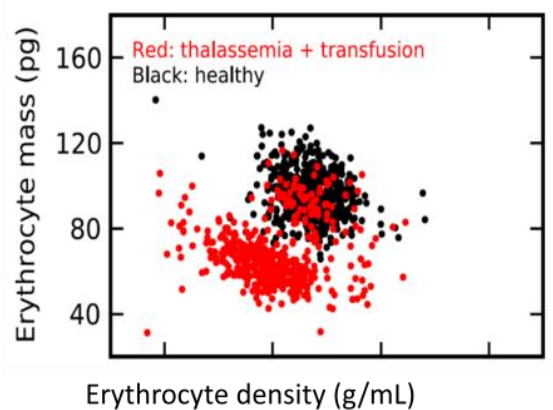

Figure 9. Suspended microchannel resonators (SMR) picture and 70 schemes showing the process to measure cell buoyant mass, the absolute mass, volume, and density of the cell passing through the inside of the device. (B) Erythrocyte mass vs. density studied by the method in (A). Erythrocytes from normal (nonthalassemic) blood and erythrocytes from a patient with thalassemia can be differentiated. Figures adapted from 75 PNAS, 2011, 108, 10992.

(SMR) have resolved the mass of single bacteria and nanomolar concentrations of proteins. Further reduction of the size of suspended microchannel resonators is expected to provide resolution to weigh single viruses and large biomolecules ${ }^{104,105}$ 80 and nanoscale SMRs have been already fabricated and tested ${ }^{106}$. Flow through detection and affinity based capture are the two strategies used in this design.

Grover et al. ${ }^{107}$ have applied their suspended microchannel resonators (SMR) to measure the buoyant mass of a cell in two 85 fluids of different densities. In their method, measurement starts with the cantilever filled with any buffer or media less dense than the cell, see figure 9 (red, step 1). The density of the red fluid is determined from the baseline resonance frequency of the cantilever. When the cell passes through the cantilever (step 2), 90 the buoyant mass of the cell in the red fluid is calculated from the peak shift in the resonance frequency. The direction of fluid flow is then reversed, and the resonance frequency of the cantilever drops as the cantilever fills with a fluid more dense than the cell (blue, step 3). The buoyant mass of the cell in the blue fluid is 
measured as the cell transits the cantilever a second time (step 4). From these four measurements of fluid density and cell buoyant mass, the absolute mass, volume, and density of the cell can be calculated.

5 In this way, they differentiated erythrocytes from thalassemic blood from healthy erythrocytes. Figure 9B represents single erythrocyte mass vs. density for an individual with suspected thalassemia trait who also received a transfusion of normal (nonthalassemic) blood 4 days prior to collection (red points, 10 figure 9B) compared to a random nonthalassemic, nontransfused individual (black points, figure 9B). The patient's own
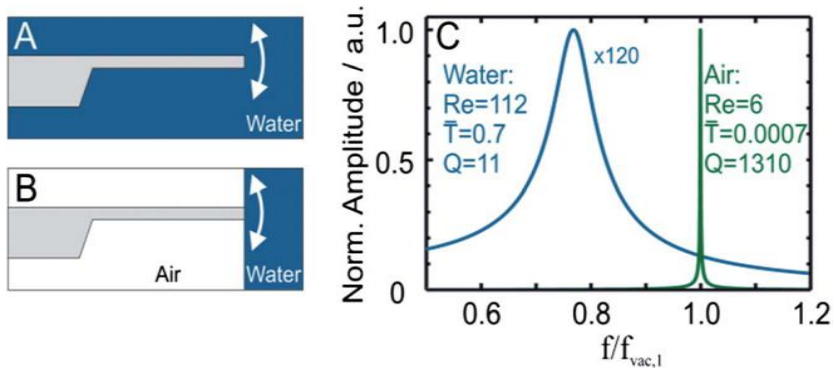

Figure 10. The concept of partial wetting for quality factor and sensitivity improvement. The quality factor in the configuration in $\mathrm{B}$ is approximately that of the same cantilever in air, and 120 times the Q of 15 the same cantilever when fully immersed in liquid. Figure from Lab on a Chip 12, 2012, 1316.

erythrocytes (red) are offset from a normal patient's erythrocytes (black), except for a small number of normal erythrocytes the thalassemic patient received during the transfusion (red points 20 clustered on black points).

These devices add to the throughput from the measurement of a large array of sensors, the capability to continuously run sample solutions containing the analyte particles, so analytes can be measured sequentially as they travel through the device inside 25 (figure 9), similarly to flow cytometry.

\subsection{Micropillar resonators}

A novel architecture dissimilar to the classic beam-like nanomechanical sensor has been recently proposed. The micropillar-resonators are a smart design that enables easy 30 sensitization at the same time as mass resolution is preserved. Kehrbusch et al. proposed the fabrication of high frequency silicon columnar microresonators that gave resonant frequencies of the lowest flexural mode of 3-7 MHz with quality factors of up to 2500 in air and 8800 under vacuum conditions. These 35 columnar microresonators demonstrated a responsivity of $1 \mathrm{~Hz} / \mathrm{fg}$ and a mass detection limit of $25 \mathrm{fg}^{108}$. They have recently proposed partial wetting (Figure 10) as a route to improve the quality factor of microresonators, both micropillars ${ }^{109}$ and microcantilevers ${ }^{110}$, in fluids by almost two orders of magnitude

40 In parallel, Melli et al ${ }^{111}$ have demonstrated this technology by investigating the formation of a single-strand DNA selfassembled monolayer (SAM) consisting of less than $10^{6}$ DNA molecules on each micropillar and by measuring their hybridization efficiency. It is remarkable that they find the 45 binding rate is 1000 times faster than on the surface of a typical microcantilever. Also, the hybridization efficiency of a SAM of maximum density DNA is $40 \%$ which is 4 times the value reported in the literature for dense layers ${ }^{111}$. They show that a regular matrix of pillars may result in a super hydrophobic 50 surface. In this configuration only the top surface of the pillar is in contact with the analyte solution while the pillar walls are not exposed, as shown in Figure 11. They were able to weigh $1.5 \mathrm{fg}$, equivalent to $7 \times 10^{5}$ DNA molecules.

The micropillar design facilitates the differential sensitization 55 of large arrays of sensors by ink-jet technologies and high multiplexing capability is expected. High throughput read-out has not yet been demonstrated with micropillars, but scanning optical read-out of large arrays could be attained by focusing the laser beam on the top of the micropillars as in reference [111] or on the
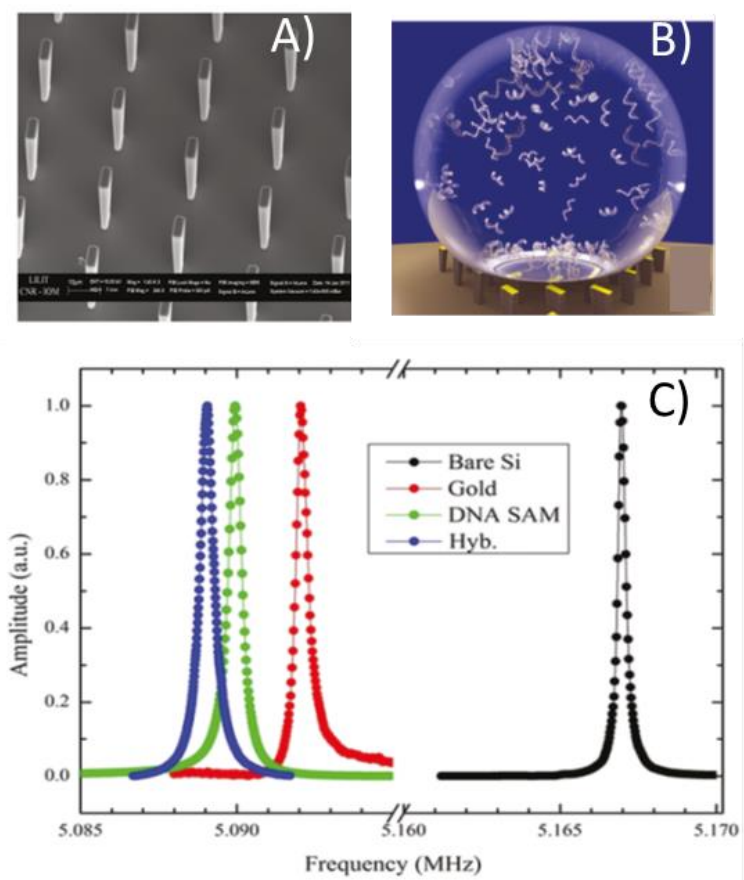

${ }_{60}$ Figure 11. (A) SEM images of the micropillars from reference [111]. (B) Schematics of superhydrophobic conditions. A drop of a solution of DNA and buffer floats on top of the pillars, and the molecules can reach only the microsized area at the end of the contact pillars. (C) Normalized resonance curves of a pillar after each experimental step, that is, bare 65 silicon (starting condition), gold coating, DNA-MCH SAM, hybridization. Adapted with permission from ACS Nano 5, 2011, 7928. Copyright 2011. American Chemical Society.

bottom side of a membrane were the pillars are fabricated on, as proposed in reference [109].

\section{9. Measuring more than mass: mechanical biomarkers}

Nanomechanical sensors have the advantage that they can be easily designed to fit the adsorbate size. Thus, for the commonly used read-out schemes, a simple change in the design of the 75 disposable sensor means the method can be applied to targets as varied in size and properties as cells, proteins or nucleic acids. This is not accessible to any other biosensing scheme. It also provides an easy way to tune the capture area to the size of the intended analyte in the way that few or single targets can be 80 analyzed by a single sensor. The thickness of the commercial cantilever sensors is of about 1 micrometer, this is approximately the size of a bacteria and one tenth the size of a white blood cell. 
The smallest silicon resonator, a nanowire, has a diameter of few tens of nanometers which is close to the size of an antibody and smaller than most viruses $(20-100 \mathrm{~nm})$. Figure 12 (A) shows the thickness of a commercial microcantilever measured by scanning 5 electron microscopy (SEM) and in (B) a silicon nanowire also measured by SEM, approximately to scale with cartoons of several representative biological targets, from a large monocyte of $10 \mu \mathrm{m}$ in diameter to an oligonucleotide of less than $10 \mathrm{~nm}$ in length.

10 The size matching, either intended or casual, it also complicates the interpretation of the response signals in the dynamic mode. When the size of the adsorbate is close to the size
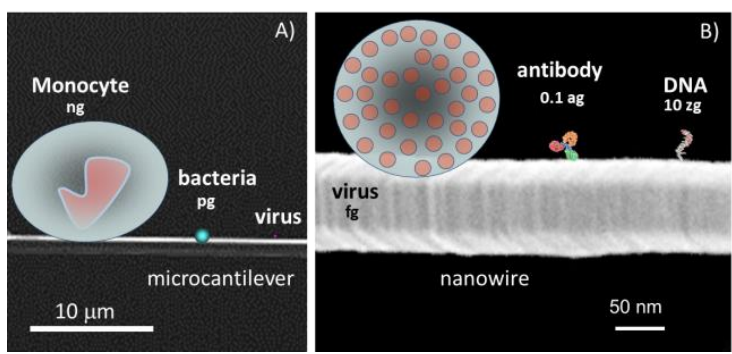

Figure 12. A) SEM image of one commercial microcantilever (thickness is shown) compared with cartoons to scale of several bioanalytes. B) 15 SEM image of a portion of one silicon nanowire grown by VLSI and shown together with cartoons to scale of several bioanalytes.

of the resonator, the mass is no longer the only parameter that affects the measured frequency shift response. Adsorbate thickness and stiffness both affect the vibration. This 20 complication has its positive counterpart, as it opens up a new route for nanomechanical sensing based on stiffness characterization $^{112-115}$.

Nanomechanical vibrations are optimally suited to listen to biological systems and to characterize their mechanical 25 properties. Again the AFM arrives as an inspiration to the field. Recently, AFM has demonstrated the classification of healthy and cancer cells by their rigidity ${ }^{116,117}$. The fact that biophysical and biomechanical properties of cells and subcellular structure influence and are influenced by onset and progression of human 30 diseases is attracting the physiologists attention ${ }^{118}$. Also, mechanical stress is considered as one of the most influential physical factors in biology, and at the same time, one of the least characterized, therefore, nanomechanical sensors in the static mode can offer a needed tool.

35 For the nanomechanical sensors to have an impact in this area, one should take into account that the contact area of the adsorbate may affect the shift due to Young's modulus. Also, the mass effect should be disentangled to the stiffness effect either by wise design of the sensors or by a correct mathematical 40 deconvolution ${ }^{22,113,119}$ Adsorbate position along the resonator is also known to affect the measurements ${ }^{112-115,119-120}$. The measurement of the quality factor together the frequency shifts has also proven useful to follow stiffness changes and to disentangle them from the mass measurement, as demonstrated 45 by $\mathrm{R}$. Gruter et $\mathrm{al}^{121}$. The design of cantilevers with adsorption sites either at the free oscillating end or at the clamped region has been used to detect DNA hybridization through changes in stiffness ${ }^{114}$ (Figure 13) and to measure the Young's modulus of E.Coli bacteria ${ }^{114}$ but this application field of nanomechanical
50 sensing is still in its infancy.

The miniaturized version of the resonators has also shown potential in the search for mechanical properties of the adsorbates. Ramos et $\mathrm{al}^{113}$ have studied nanowires under nonpunctual mass adsorption. They propose a sensing paradigm 55 based on the degeneration breakage of the NW vibration modes. The method allows measuring the mass with ultra-high sensitivity and, in addition, classifying the adsorbate on the basis of its mechanical properties (Figure 14). These experiments are based on three measurements easily accessible in most experimental 60 set-ups: eigenfrequency sum, eigenfrequency difference and amplitude (orientation of the vibration planes). They show that
A)

A) $\mathrm{MCH}$

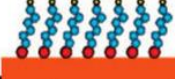
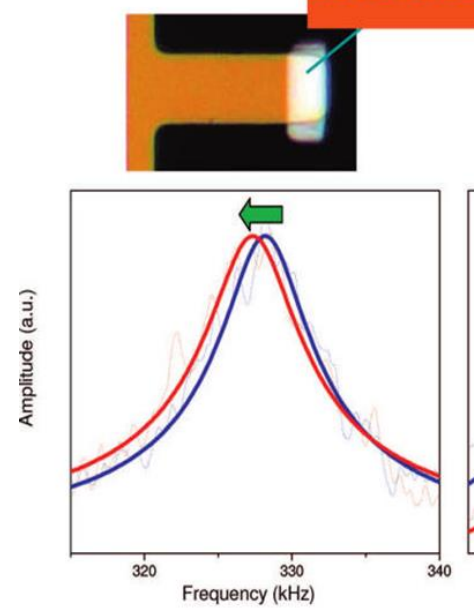

B)
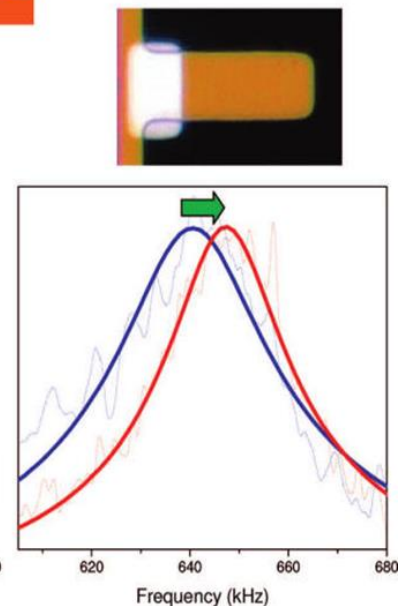

Figure 13. Representative resonance peaks of cantilevers with the receptor gold area at the tip (A) and at the clamped end (B) before (blue line) and after adsorption of a mercaptohexanol $(\mathrm{MCH})$ monolayer (red 65 dashed peaks). Cantilevers were designed to disentangle the mass effect (negative shift in (A)) and the stiffness effect (positive shift in (B)). Optical images of the cantilevers and schematic of the $\mathrm{MCH}$ selfassembled monolayer are also show on top of the graphs. Cantilevers are $15 \mu \mathrm{m}$ long, $6 \mu \mathrm{m}$ wide and $100 \mathrm{~nm}$ thick. With permission from Anal. 70 Chem. 2009, 81, 2274.

for set-ups where adsorption is implemented coming from multiple sources, as could be the case for future nanomechanical mass spectrometers, the technique can discriminate the source on basis of the deposition angle. They demonstrate zeptogram mass 75 sensitivity and discrimination in variations in the Young's modulus of about $0.1 \mathrm{kPa}$ per femtogram of sample. Since dry proteins have a Young's modulus in the 0.1-10 GPa range, the proposed technique could detect variations of 100 parts per million in the Young's modulus of a single protein. This 80 unmatched capability of resonant NWs for measuring mechanical properties in addition to mass with ultrahigh sensitivity opens the door for relevant biomedical applications. It is increasingly clear the important role of the mechanical properties in biological processes and pathogenic disorders, therefore nanoresonators 85 could provide a unique tool in this field of research. As a paramount example, a single point mutation in the capsid protein of some viruses can significantly change the stiffness of the virus particle $^{122}$ and thus, its invasiveness.

\section{Summary and future perspective}


The nanomechanical sensors have now a promising horizon for their application to relevant problems in Biology. They are manageable, easy to sensitize, require few chemistry steps and have high intrinsic sensitivity. We lack from systematic studies 5 and large numbers of measurements in relevant biological applications, far from the proof of concept approach pursued to date, but the technology to attain this goal is already at reach. Multiple probe measurements, including statistical evaluation of acquired data, will be the key to find nanomechanical sensors in 10 every biochemistry laboratory in the near future. The beauty of nanomechanical sensors is that they are best suited to listen to diverse parameters of biological systems. Therefore, following
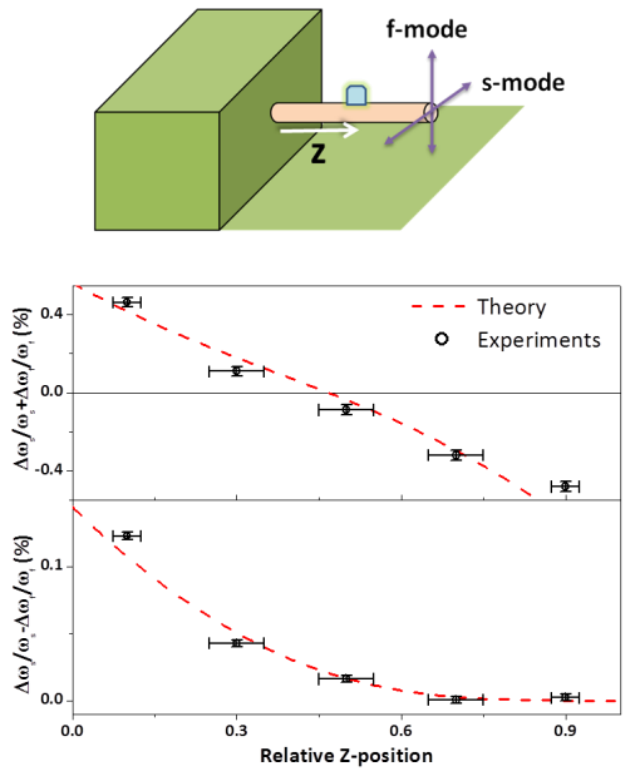

Figure 14. Effect of mass deposition position on frequency splitting. (A) Plots show the sum (top) and difference (bottom) of the relative frequency 15 shifts of a one-dimensional nanowire resonator as a function of the position $\mathrm{z}$ at which a mass of $3 \mathrm{fg}$ is deposited on the nanowire along the fast-mode vibration axis (f-mode). The symbols are experimental data and the dashed red line is a theoretical prediction based on the Ritz method applied to the beam equation in two dimensions as described in reference 20 [113]. The mass and mechanical properties of the adsorbate can be determined by measuring the sum and difference of the relative frequency shifts. (B) Scheme of non-punctual mass adsorption on a silicon nanowire indicating decomposition of its vibration in the fast mode (higher frequency) and slow mode (lower frequency). Reprinted with permission 25 from Nature Nanotechnology, 2010, 5, 641.

the mass, the intermolecular forces, structural changes and the stiffness of bioanalytes, as shown in this review, are presumably not the only future routes for the technology. We foresee further innovative ways to take advantage of nanomechanics for 30 biosensing in the coming years.

\section{Acknowledgements}

The authors would like to thank E. Gil-Santos, F. N. Martínez and S. González for fruitful discussion. We acknowledge financial support from the Spanish Science Ministry through 35 projects ANEM! (TEC2009-14517-C02), INMUNO-swing (IPT2011-0821-010000) and ForceForFuture (CSD2010-00024); and from European Research Council through Starting Grant NANOFORCELLS (ERC-StG-2011-278860).

\section{Notes and references}

40 Address, Institute of Microelectronics of Madrid, CSIC, Isaac Newton 8 (PTM), Tres Cantos, 28760 Madrid, Spain

Fax: +34 918060701Tel: +34 918060700;

mcalleja@imm.cnm.csic.es

\section{${ }_{45}$ References}

1. J. R. Barnes, R. J. Stephenson, M. E. Welland, CH. Gerber and J. K. Gimzewski, Nature, 1994, 372, 79

2. T. Thundat, E. A. Wachter, S. L. Sharp and R. J. Warmack, 1995. Appl. Phys. Lett, 1995, 66, 1695

50 3. J. Fritz, M. K. Baller, H. P. Lang, H. Rothuizen, P. Vettiger, E Meyer, H. J. Güntherodt, Ch. Gerber and J. K. Gimzewski, Science, 2000, 288, 316

4. S. Fanget, S. Hentz, P. Puget, J. Arcamone, M. Matheron, E. Colinet, P. Andreucci, L. Duraffourg, E. Myers and M. L. Roukes, Sens.

55 Actuator B-Chem, 2011, 160, 804

5. S. Lenz, S. K. Nett, M. Memesa, R. F. Roskamp, A. Timmann, S. V. Roth, R. Berger and J. S. Gutmann, Macromolecules, 2010, 43, 1108

6. S. Lenz, A. Ruhm, J. Major, R. Berger and J. S. Gutmann, Macromolecules, 2011, 44, 360

607 . M. Toda, T. Fujii, A. Yoshida, T. Hashida and T. Ono, Appl. Phys. Lett., 2011, 99, 074101

8. J. W. Ndieyira, M. Watari, A. D. Barrera, D. Zhou, M. Vogtli, M. Batchelor, M. A. Cooper, T. Strunz, M. A. Horton, C. Abell, T. Rayment, G. Aeppli and R. A. McKendry, Nature Nanotech., 2008,

$65 \quad 3,691$

9. J. Zhang, H. P. Lang, F. Huber, A. Bietsch, W. Grange, U. Certa, R. Mckendry, H. J Günteherodt, M. Hegner and C. Gerger, Nature Nanotech., 2006, 1, 214

10. G. Wu, R. H. Datar, K. M. Hansen, T. Thundat, R. J. Cote and A. Majumdar, Nature Biotech., 2001, 19, 856

11. P. S. Waggoner, C. P. Tan and H. G. Craighead, Sens. Actuator B Chem, 2010, 150, 550

12. M. Varshney, P. S. Waggoner, C. P. Tan, K. Aubin, R. A. Montagna and H. G. Craighead, Anal. Chem., 2008, 80, 2141

75 13. B. Ilic, Y. Yang and H. G. Craighead, Appl. Phys. Lett., 2004, 85, 2604

14. E. Timurdogan, B. E. Alaca, I. H. Kavakli and H. Urey, Biosens. Bioelectron., 2011, 28, 189

15. K. Gruber, T. Horlacher, R. Castelli, A. Mader, P. H. Seeberger and B. A. Hermann, ACS Nano, 2011, 5, 3670

16. M. Inomata, M. Toda, M. Sato, A. Ishijima and T. Ono, Appl. Phys. Lett., 2012, 100, 154104

17. K. R. Buchapudi, X. Huang, X. Yang, H. F. Ji and T. Thundat, Analyst, 2011, 136, 1539

85 18. P. S. Waggoner and H. G. Craighead, Lab Chip, 2007, 7, 1238

19. S. Xu and R. Mutharasan R, Expert Opin. Drug Dis., 2009, 4, 1237

20. J. L. Arlett, E. B. Myers and M. L. Roukes, Nature Nanotech., 2011, 6, 203

21. A. Boisen, S. Dohn, S. S. Keller, S. Schmid and M. Tenje, Rep. Prog. $90 \quad$ Phys., 2011, 74, 036101

22. K. Eom, H. S. Park, D. S. Yoon and T. Kwon, Phys. Rep., 2011, 503, 115

23. M. Godin, V. Tabard-Cossa, Y. Miyahara, T. Monga, P. J. Williams, L. Y. Beaulieu, R. B. Lennox and P. Grutter Nanotechnology, 2010, 21, 075501

24. H. Craighead, Nature Nanotech., 2007, 2, 18

25. P. S. Waggoner, M. Varshney and H. G. Craighead, Lab Chip, 2009, 9, 3095

26. R. McKendry, J. Y. Zhang, Y. Arntz, T. Strunz, M. Hegner, H.P. 100 Lang, M.K. Baller, U. Certa, E. Meyer, H. J. Güntherodt and C. Gerber, Proc. Natl. Acad. Sci., 2002, 99, 9783

27. M. Yue, H. Lin, D.E. Dedrick, S. Satyanarayana, A. Majumdar, A. S. Bedekar, J. W. Jenkins, S. Sundaram, J. Microelectromech Systems 13, 290 (2004)

105 28. T. Braun, V. Barwich, M. K. Ghatkesar, A. H. Bredekamp, C. Gerber, M . Hegner, and H. P. Lang, Phys. Rev. E, 2005, 72, 031907 
29. P. A. Truitt, J. B. Hertzberg, C. C. Huang, K. L Ekinci and K. C. Schwab, Nano Lett., 2007, 7, 120

30. J. Arcamone, A. Niel, V. Gouttenoire, M. Petitjean, N. David, R. Barattin, M. Matheron, F. Ricoul, T. Bordy, H. Blanc, J. Ruellan, D. Mercier, N. Pereira-Rodriguez, G. Costa, V. Agache, S. Hentz, J. Gabriel, F. Baleras, C. Marcoux, T. Ernst, L. Duraffourg, E. Colinet, E. B. Myers, M. L. Roukes, P. Andreucci, E. Ollier and P. Puget, IEDM, 2011, 29.3.1

31. M . Li, E. B. Myers, H. X. Tang, S. J. Aldridge, H. C. McCaig, J. J.

10 Whiting, R. J. Simonson, N. S. Lewis and M. L. Roukes, Nano Lett., 2010, 10, 3899

32. A. Sampathkumar and K. L. Ekinci, T. W. Murray, Nano Lett., 2011, 11, 1014

33. N. F. Martinez, P. M. Kosaka, J. Tamayo, J. Ramirez, O. Ahumada,

15 J. Mertens, T. D. Hien, C. V. Rijn and M. Calleja, Rev. Sci. Instrum., 2010, 81, 125109

34. I. Bargatin, E. B. Myers, J. S. Aldridge, C. Marcoux, P. Brianceau, L. Duraffourg, E. Colinet, S. Hentz, P. Andreucci, M. L. Roukes, Nano Letters, 2012, 12, 1269

20 35. P. Ivaldi, J. Abergel, M. H. Matheny, L. G. Villanueva, R. B. Karabalin, M. L. Roukes, P. Andreucci, S. Hentz and E. Defay, $J$. Micromech. Microeng., 2011, 21, 085023

36. M. Favre, J. Polesel-Maris, T. Overstolz, P. Niedermann, S. Dasen, G. Gruener, R. Ischer, P. Vettiger, M. Liley, H. Heizelmann and A. Meister, J. Mol. Recogn., 2011, 24, 446

37. J. Polesel-Maris, L. Aeschimann, A. Meister, R. Ischer and E. Bernard, ICONN, 2007, 61, 955

38. P. Vettiger, T. Albrecht, M. Despont, U. Drechsler, U. Durig,B. Gotsmann, D. Jubin, W. Haberle, M. A. Lanz, H. Rothuizen, R. Stutz, D. Wiesmann G: K. Binning, P. Bachtold, G: Cherubini, C. Hagleitner, T. loeliger, A. Pantazi H. Pozidis and E. Eleftheriou, IEDM, 2003, 32.1.1

39. E. Eleftheriou, T. Antonakopoulos, G. K. Binnig, G. Cherubini, M. Despont, A. Dholakia, U. Durig, M. A. Lantz, H. zidis, H. F.

35 Rothuizen and P. Vettiger, IEEE Trans. Magn., 2003, 39, 938

40. www.concentris.com

41. www.mecwins.com

42. T. Braun, M. K. Ghatkesar, N. Backmann, W. Grange, P. Boulanger, L. Letellier, H. P. Lang, A. Bietsch, C. Gerber and M. Hegner,

$40 \quad$ Nature Nanotech., 2009, 4, 179

43. A. Bietsch, J. Y. Zhang, M. Hegner, H. P. Lang and C. Gerber, Nanotechnology, 2004, 15, 873

44. T. Thorsen, S. J. Maerkl and S. R. Quake, Science, 2002, 298, 580

45. A. Johansson, M. Calleja, P. A. Rasmussen and A. Boisen, Sens.

45 Actuators A. Phys., 2005, 123, 111

46. F. G. Bosco, E. T. Hwu, C. H. Chen, S. Keller, M. Bache, M. H, Jakobsen, I. S. Hwang and A. Boisen, Lab Chip, 2011, 11, 2411

47. L. A. Pinnaduwage, A. C. Gehl, S. L. Allman, A. Johansson and A. Boisen, Rev. Sci. Instrum., 2007, 78, 055101

50 48. G. Yoshikawa, H. P. Lang, T. Akiyama, L. Aeschimann, U. Staufer, P. Vettiger, M. Aono, T. Sakurai and C. Gerber, Nanotechnology, 2009, 20, 015501

49. G. Shekhawat, S. H. Tark, V. P. Dravid, Science, 2006, 311, 1592

50. H. P. Lang, R. Berger, C. Andreoli, J. Brugger, M. Despont, P.

55 Vettiger, C. Gerber, J. K. Gimzewski, J. P. Ramseyer, E. Meyer and H. J. Güntherodt, Appl. Phys. Lett., 1998, 72, 383

51. F. M. Battiston, J. P. Ramseyer, H. P. Lang, M. K. Baller, C. Gerber, J. K. Gimzewski, E- Meyer and H. J. Güntherodt, Sens. Actuators B Chem., 2001, 77. 122

60 52. W. S. Hu, R. Anderson, Y. S. Qian, J. G. Song, J. W. Noh, S. Kim, G. P. Nordin, Rev. Sci. Instrum.,2009, 80, 085101

53. M. Nordstrom, D. A. Zauner, M. Calleja, J. Hubner, A, Boisen, Appl. Phys. Lett., 2007, 91, 103512

54. M. Alvarez and J. Tamayo, Sens. Actuators B Chem., 2005, 106, 687

${ }_{65}$ 55. P. M. Kosaka, J. Tamayo, E. Gil-Santos, J. Mertens, V. Pini, N. F. Martínez, O. Ahumada and M. Calleja, J. Appl. Phys., 2011, 109, 064315

56. J. Mertens, M. Alvarez and J. Tamayo, Appl. Phys. Lett., 2005, 87, 234102

70 57. J. Tamayo, V. Pini, P. Kosaka, N. F. Martinez, O. Ahumada and M. Calleja, Nanotechnology,.
58. Y. W. Lai, S. Hamann, M. Ehmann and A. Ludwig, Rev. Sci. Instrum, 2011, 82, 063903

59. J. Reed, J. Schmit, S. Han, P. Wilkinson and J. K. Gimzewski, Opt. Laser Eng., 2009, 47, 217

60. J. Wehrmeister, A. Fuss, F. Saurenbach, R. Berger and M. Helm, Rev. Sci. Instrum, 2007, 78, 104105

61. S. Kelling, F. Paoloni, J. Z. Huang, V. P. Ostanin, S. R. Elliott, Rev. Sci. Instrum, 2009, 80, 093101

80 62. J. Schmit, J. Reed, E. Novak and J. K. Gimzewski, J. Opt. A-Pure Appl. Op., 2008, 10, 064001

63. M. Spletzer, A. Raman, H. Sumali and J. P. Sullivan, Appl. Phys. Lett., 2008, 92, 3

64. J. N. Murphy, A. K. H. Cheng, H. Z. Yu and D. Bizzotto, J. Am. Chem. Soc., 2009, 131, 4042

65. M. Yue, J. C. Stachowiak, H. Lin, R. Datar, R. Cote and A. Majumdar, Nano Lett., 2008, 8, 520

66. M. L. Sushko, J. H. Harding, A. L. Shluger, R. A. McKendry and M. Watari, Adv. Mater., 2008, 20, 3848

90 67. J. A. Swets, Science, 1988, 240, 1285

68. T. Fawcet, Pattern Recognition Letters, 2006, 27, 86169.

69. P. Kosaka, J. Tamayo, J.J. Ruz, S. Puertas, E. Polo, V. Grazu, J. M de la Fuente and M. Calleja, submitted

70. J. Joo, D. Kwon, C. Yim, S. Jeon, ACS Nano, 2012, 6, 4375

95 71. M. K. Ghatkesar, H. P. Lang, C. Gerber, M. Hegner and T. Braun, PLoS One, 2008, 3, e3610

72. W. M. Shu, D. S. Liu, M. Watari, C. K. Riener, T. Strunz, M. E. Welland, S. Balsubramanian and R. A. Mckendry, J. Am. Chem. Soc., 2005, 127, 17054

100 73. J. Mertens, J. Tamayo, P. Kosaka and M. Calleja, Appl. Phys. Lett., 2011, 98, 153704

74. J. Mertens, C. Rogero, M. Calleja, D. Ramos, J. A. Martin-Gago, C. Briones and J. Tamayo, Nature Nanotech., 2008, 3, 301

75. M. Wagman, S. Medalion and Y. Rabin, Soft Matter, submitted

105 76. J. Mertens, M. I. Dauden, J. L. Carrascosa and J. Tamayo, Nanotechnology, 2012, 23, 015501

77. K. Eom, H. Jung, G. Lee, J. Park and K. Nam, ChemmComm, 2012, 48, 955

78. A. K. Naik, M. S. Hanay, W. K. Hiebert, X. L. Feng and M. L. Roukes, Nature Nanotech., 2009, 4, 445

79. J. Park, H. Qin, M. Scalf, R. T. Hilger, M. S. Westphall, L. M. Smith and R. H. Blick, Nano Lett., 2011, 11, 3681

80. G. C. Hilton, J. M. Martinis, D. A. Wollman, K. D. Irwin, L. L. Dulcie, D. Gerber, P. M. Gillevet and D. Twerenbold, Nature, 1998, 391, 672

81. J. Chaste, M. Sledzinska, M. Zdrojek, J. Moser and A. Bachtold, Appl. Phys. Lett., 2011, 99, 213502

82. A. Eichler, J. Chaste, J. Moser and A. Bachtold, Nano Lett., 2011, 11, 2699

120 83. A. Eichler, J. Moser, J. Chaste, M. Zdrojek, I. Wilson-Rae and A. Bachtold, Nature Nanotech., 2011, 6, 339

84. K. Jensen, K. Kim and A. Zettl, Nature Nanotech., 2008, 3, 533

85. J. Chaste, A. Eichler, J. Moser, G. Ceballos, R. Rurali and A. Bachtold, Nature Nanotech, 2012, 7, 301

125 86. D. Ramos, E. Gil-Santos, V. Pini, J. M. Llorens, M. FernandezRegulez, A. SanPaulo, M. Calleja and J. Tamayo, Nano Lett.,2012, 12, 932

87. M. W. Li, R. B. Bhiladvala, T. J. Morrow, J. A. Sioss, K. K. Lew, J. M. Redwing, C. D. Keating and T. S. Mayer, Nature Nanotech., 2008, 3, 88

88. J. Arcamone, E. Dujardin, G. Rius, F. Pérez-Murano and T. Ondarçuhu, J. Phys. Chem. B, 2007, 111, 13020

89. A. San Paulo, R. T. Howe, J. Bokor, R. He, P. Yang, D. Gao, C. Carraro and R. Maboudian, Appl. Phys. Lett., 2005, 87, 53111

135 90. R. R. He, X. L. Feng, M. L. Roukes and P. D. Yang, Nano Lett.,2008, 8, 1756

91. L. G. Villanueva, R. B. Karabalin, M. H. Matheny, E. Kenig, M. C. Cross and M. L. Roukes, Nano Lett.,2011, 11, 5054

92. W. H. Zhang, R. Baskaran, K. L. Tumer, Sens. Actuator A Phys., 2002, 102, 139

93. G. A. Campbell and R. Mutharasan, Anal. Chem., 2006, 78, 2328

94. G. A. Campbell and R. Mutharasan, Anal. Chem., 2007, 79, 1145 
95. B. N. Johnson and R. Mutharasan, Biosens. Bioelectron., 2012, 32, 1

96. A. Cagliani and Z. J. Davis, J. Micromech. Microeng., 2011, 21, 045016

97. S. R. Hong, H. D. Jeong and S. Hong, Talanta, 2010, 82, 899

5 98. M. Spletzer, A. Raman, A. Q. Wu, X. F. Xu and R. Reifenberger, Appl. Phys. Lett., 2006, 88, 254102

99. E. Gil-Santos, D. Ramos, A. Jana, M. Calleja, A. Raman and J. Tamayo, Nano Lett., 2009, 9, 4122

100.E. Gil-Santos, D. Ramos, V. Pini, M. Calleja and J. Tamayo, Appl. Phys. Lett., 2011, 98, 123108

101.P. Thiruvenkatanathan, J. Woodhouse, J. Yan and A. A. Seshia, J. Appl. Phys., 2011, 109, 104903

102.H. Pakdast and M. Lazzarino, Sens. Actuator A Phys., 2012, 175. 127

103.M. K. Ghatkesar, V. Barwich, T. Braun, J. P. Ramseyer, C. Gerber,

15 M. Hegner, H. P. Lang, U. Drechsler and M. Despont, Nanotechnology, 2007, 18, 445502

104.J. E. Sader, T. P. Burg, J. Lee and S. R. Manalis, Phys. Rev. E, 2011, 84, 026304

105.T. P. Burg, J. E. Sader and S. R. Manalis, Phys. Rev. Lett., 2009, 102, $20 \quad 228103$

106.R. A. Barton, B. Ilic, S. S. Verbridge, B. R. Cipriany, J. M. Parpia and H. G. Craighead, Nano Lett.,2010, 10, 2058

107.W. H. Grover, A. K. Bryan, M. Diez-Silva, S. Suresh, J. M. Higgins and S. R. Manalis, PNAS, 2011, 108, 10992

25 108.J. Kehrbusch, E. A. Ilin, M. Hullin and E. Oesterschulze, Appl. Phys. Lett., 2008, 93, 023102

109.E. Oesterschulze, P. Kehrbusch, B. Radzio, E. A. Ilin and A. Thyssen,J. W. Deitmer and J. Kehrbusch, Lab Chip , 2012, 12, 1316

110. J. Linden, E. Oesterschulze, Appl. Phys. Lett., 2012, 100, 113511

30 111.M. Melli, G. Scoles and M. Lazzarino, ACS Nano, 2011, 15, 7928

112.J. Tamayo, D. Ramos, J. Mertens and M. Calleja, Appl. Phys. Lett., 2006, 89, 224104

113.E. Gil-Santos, D. Ramos, J. Martinez, M. Fernandez-Regulez, R. Garcia, A. San Paulo, M. Calleja and J. Tamayo Nature Nanotech., 2010, 5, 641

114.D. Ramos, M. Arroyo-Hernandez, E. Gil-Santos, H. D. Tong, C. Van Rijn, M. Calleja and J. Tamayo, Anal. Chem., 2009, 81, 2274

115.D. Ramos, J. Tamayo, J. Mertens, M. Calleja and A. Zaballos, J. Appl. Phys., 2006, 100, 106105

40 116.S. E. Cross, Y. S. Jin, J. Rao and J. K. Gimzewski, Nature Nanotech., 2007, 2, 780

117.A. Raman, S. Trigueros, A. Cartagena, A. P. Z. Stevenson, M. Susilo, E. Nauman and S: Antoranz Contera, Nature Nanotech., 2011, 6, 809

118.S. Suresh, Acta Mater., 2007, 55, 3989

45 119.D. Ramos, J. Tamayo, J. Mertens, M. Calleja, L. G. Villanueva and A. Zaballos, Nanotechnology, 2008, 19, 035503

120.S. Dohn, S. Schmid, F. Amiot and A. Boisen, Appl. Phys. Lett., 2010, 97, 044103

121.R. R. Gruter, Z. Khan, R. Paxman, J. W. Ndieyira, B. Dueck, B: A.

$50 \quad$ Bircher, J. L. Yang, U. Drechsler, M. Despont, R. A. McKendry and B. W. Hoogenboom, Appl. Phys. Lett., 2010, 96, 023113

122.J. P. Michel, I. L. Ivanovska, M. M. Gibbons, W. S. Klug, C. M. Knobler, G. J. L. Wuite and C. F. Schmidt, PNAS, 2006, 103, 6184

123.B. Ilic, D. Czaplewski, H. G. Craighead, P. Neuzil, C. Campagnolo

55 and C. Batt, Appl. Phys. Lett., 2000, 77, 450 